\title{
Reversible reduction of cardiac sympathetic innervation after coronary artery bypass graft surgery: An observational study using serial iodine 123-labeled meta-iodobenzyl-guanidine (MIBG) imaging
}

Athanasios Katsikis, MD, George Ekonomopoulos, MD, Spyros Papaioannou, MD, Anna Kouzoumi, and Maria Koutelou, $\mathrm{PhD}$

Objectives: Various types of surgical and interventional procedures have been reported to cause cardiac sympathetic denervation. We aimed at evaluating the effects of coronary artery bypass grafting (CABG) in cardiac sympathetic innervation through meta-iodobenzyl-guanidine (MIBG) imaging.

Methods: MIBG imaging was performed in 21 patients with coronary artery disease (CAD) 1 day before and 1 week and 6 months after CABG with concomitant measurements of corrected QT interval. In each study we evaluated MIBG defect score in a 16-segment left ventricular model, MIBG-defect size (percent) from generated polar maps, and heart/mediastinum ratio.

Results: Mean MIBG defect score and size were increased ( $32 \pm 9.5$ vs $24 \pm 5, P<.0001$, and $49.5 \% \pm 20.4 \%$ vs $37 \% \pm 8.7 \%, P=.004$, respectively) and mean heart/mediastinum ratio was reduced ( $1.5 \pm 0.4$ vs $1.9 \pm 0.3$, $P<.0001)$ at 1 week after CABG. At 6 months these indices had no significant differences compared with their pre-CABG values. Mean corrected QT interval demonstrated no significant changes. Increase in MIBG score in the second imaging was associated with adverse events related to arrhythmia and myocardial dysfunction during the 6-month follow-up period in a binary logistic regression model.

Conclusions: $\mathrm{CABG}$ is associated with clinically important but reversible reduction in cardiac sympathetic nerve function, with periprocedural effects (cardioplegia, hypothermia, ischemia, direct nerve injury) being possible mechanisms for this finding. (J Thorac Cardiovasc Surg 2012;144:210-6)

Supplemental material is available online.

The autonomic nervous system plays a major role in the regulation of cardiac function and many cardiac disorders involve alterations of cardiac adrenergic neurotransmission. ${ }^{1}$

Meta-iodobenzyl-guanidine (MIBG) is an analog of the adrenergic blocking agent guanethidine and follows the same active uptake, storage, and release pathways in the sympathetic nerve endings as does norepinephrine.

MIBG labeling with iodine $123\left({ }^{123} \mathrm{I}\right)$ allows for imaging; thus quantitative ${ }^{123}$ I-MIBG imaging is a direct, noninvasive, in vivo method of assessment of global and regional sympathetic integrity, by measurement of MIBG uptake, ${ }^{2}$ and function, by measurement of MIBG washout.

From the Onassis Cardiac Surgery Center, Athens, Greece.

Disclosures: Authors have nothing to disclose with regard to commercial support.

Received for publication June 19, 2011; revisions received Jan 25, 2012; accepted for publication March 12, 2012; available ahead of print April 9, 2012.

Address for reprints: Athanasios Katsikis, MD, Zoodochou Pigis 54, Melissia,

Athens, Greece (E-mail: tkatsikis@ hotmail.com).

0022-5223/\$36.00

Copyright (c) 2012 by The American Association for Thoracic Surgery

doi:10.1016/j.jtcvs.2012.03.005
Diminished MIBG uptake has been related to reduction in cardiac sympathetic nerve function (CSNF) in the setting of various heart diseases. ${ }^{3-5}{ }^{123}$ I-MIBG imaging has provided important insights to the pathophysiologic mechanisms of these disorders with good evidence for prognostic significance in many cases. ${ }^{6,7}$

There is evidence that reduction in CSNF can also be observed after coronary artery bypass graft (CABG) surgery, but only limited data about the role of MIBG imaging in this setting exist. ${ }^{8}$

The present study examines whether CABG-induced reduction in CSNF occurs in patients with multivessel coronary artery disease (CAD), to what extent this phenomenon is reversed after $\mathrm{CABG}$, and whether it has any effects on postprocedural morbidity and mortality.

\section{METHODS}

\section{Patients}

Twenty-one patients with CAD were prospectively studied. Inclusion criteria were as follows: multivessel $\mathrm{CAD}$ amenable to revascularization and elective $\mathrm{CABG}$ procedure indicated for active angina, more than low-risk features in noninvasive testing, or 3-vessel disease in association with proximal left anterior descending artery (LAD) disease, reduced systolic function, or diabetes. Exclusion criteria were as follows: previous myocardial infarction (MI) less than 1 month before the operation, 


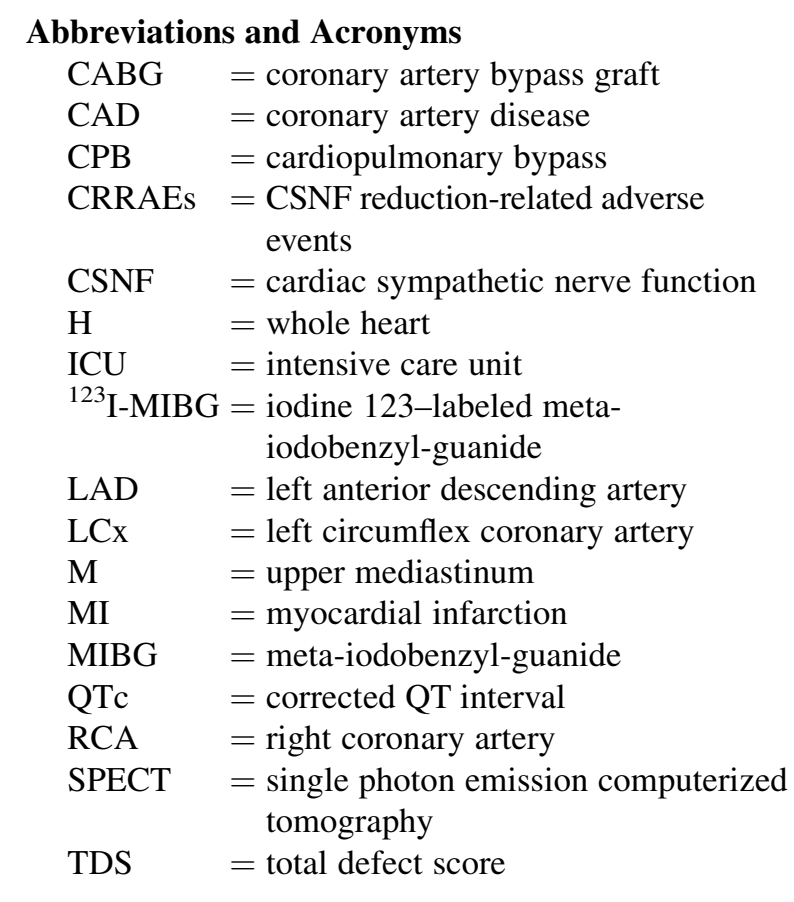

symptoms of acute coronary syndrome or positive cardiac biomarkers before $\mathrm{CABG}$, diseases of the central or peripheral nervous system, history of thyroid disease, and pre-existing renal failure.

According to these criteria, 21 consecutive, eligible patients who underwent coronary angiography in our institution from January 1 to August 1, 2008, and received a recommendation for surgical revascularization were enrolled in our study, providing written informed consent, and the study was approved by the Ethics Committee of the Onassis Cardiac Surgery Center.

Patients' characteristics are shown in Table 1. On enrollment, 19 patients were receiving beta-blockers, 15 , oral nitrates, 12 , statins, and none was receiving calcium channel blocker; all of them received aspirin. At 6 months, beta-blocker and aspirin use remained unchanged, whereas statin use had increased (16 patients) and nitrate use had decreased (1 patient). In cases in which patients were using medications known to interfere with MIBG uptake and biodistribution other than beta-blockers, these drugs were withheld for 2 days before ${ }^{123}$ I-MIBG imaging. Detailed information about patients' medications at each stage of the study can be found in Table E1.

Seven patients had a history of type II diabetes for a mean duration of $12.1 \pm 3.2$ years. Ten patients had a history of at least $1 \mathrm{MI}$, dating 4 to 228 months (median duration, 9 months) before CABG. All of these patients had at least 1 previous ST-segment elevation MI (4 infarcts in the LAD, 6 in the right [RCA], and 1 in the left circumflex [LCx] coronary artery distribution) and 3 of them also had a history of nonST-segment elevation MI, while in total there were 3 patients with multiple MIs.

\section{Study Protocol}

${ }^{123}$ I-MIBG scintigraphy was performed 1 day before and both 1 week and 6 months after cardiac surgery. At the same time points electrocardiograms were taken for determination of corrected QT interval (QTc). Furthermore, blood samples were drawn for determination of complete blood count, common biochemistry panel, and myocardial enzymes during the first 7 days of the postoperative period as a standard follow-up procedure for patients undergoing $\mathrm{CABG}$.
The second ${ }^{123}$ I-MIBG imaging took place after patients had been transported from the intensive care unit (ICU) to common hospital wards.

Aortic crossclamp time and total time of extracorporeal circulation were recorded for each patient, and postprocedural serial measurement of myocardial enzyme activity was used according to standard ICU policy for the treatment of patients after CABG. Elevations of creatine kinase $\mathrm{MB}$ and/or cardiac troponin I more than 5 times the 99th percentile of the normal reference range of our hospital laboratory during the first 72 hours after CABG were defined as periprocedural myocardial necrosis. When these elevations were combined with new pathologic Q waves, left bundle branch block, or the need for repeat revascularization in the following 7 days, a CABG-related MI was established. The definitions used were in accordance with the 2007 consensus document of ESC/ACCF/AHA/WHF regarding the universal definition of MI. ${ }^{9}$

Among the various modalities established for the assessment of autonomic nervous system function, we used the QTc interval ${ }^{10}$ for evaluation of the effects of sympathetic tone in MIBG scintigraphy findings. QTc is a reproducible, easily measurable parameter, with the advantage of not requiring patient cooperation. ${ }^{11}$

Finally, CSNF reduction-related adverse events (CRRAEs) were recorded per patient to evaluate the possible clinical significance of the observed changes in CSNF. Such events were considered (1) sustained supraventricular or ventricular arrhythmias documented between the time of postprocedural restoration of circulation and the third MIBG imaging study and (2) the need for combined pharmacologic or mechanical support because of low cardiac output syndrome during the patient's hospitalization.

\section{${ }^{123}$ I-MIBG Imaging}

All subjects received $5 \mathrm{~mL}$ of $1 \%$ Lugol solution 48 hours before and 48 hours after administration of ${ }^{123}$ I-MIBG to block tracer uptake in the thyroid gland.

We performed delayed ${ }^{123}$ I-MIBG imaging using a single-head, rotating gamma camera equipped with a low-energy, general-purpose collimator (ORBITER 75 ZLC; Siemens AG, Munich, Germany).

In the fasting state $5 \mathrm{mCi}(185 \mathrm{MBq})$ of ${ }^{123} \mathrm{I}-\mathrm{MIBG}$ (GE Healthcare, Cygne, Netherlands) was injected intravenously through an antecubital vein at rest and imaging was started 4 hours after injection for both planar and single photon emission computerized tomography (SPECT) images.

Anterior (front) planar images of the chest were initially acquired over 5 minutes in a $64 \times 64$ matrix. Immediately after planar imaging, 32 images were obtained in a $64 \times 64$ matrix for 40 seconds, with a $180^{\circ}$ rotation ranging from the $45^{\circ}$ right anterior oblique position to the left posterior oblique position. Energy discrimination was provided by a $20 \%$ window centered at $159 \mathrm{keV}$.

On the anterior planar ${ }^{123}$ I-MIBG images, regions of interest (ROIs) were drawn manually over the whole heart $(\mathrm{H})$ and upper mediastinum (M). Using the counts in the regions of interest, the H/M ratio of MIBG were calculated as follows: $\mathrm{H} / \mathrm{M}=$ mean pixel counts of heart region of interest/mean pixel counts of mediastinal region of interest. In our laboratory, the normal ${ }^{123} \mathrm{I}-\mathrm{MIBG} \mathrm{H} / \mathrm{M}$ ratio values of the delayed images obtained from age-matched controls are 1.8 to 2.7. Normal subjects consisted of 9 ( 5 men and 4 women; mean age, $59 \pm 9$ years) individuals with normal findings in the echocardiogram and low likelihood of CAD based on risk factors, history, and electrocardiogram.

After preprocessing with a Butterworth filter (cutoff frequency, 0.44 cycle/cm; power factor, 8), transaxial SPECT images were reconstructed by filtered back projection without attenuation or scatter correction and were displayed as short- and long-axis (horizontal and vertical) slices.

Regional uptake was estimated through visual interpretation of SPECT images performed by 2 experienced observers, using a 16-segment left ventricular model and a 5 -point scoring system $(0=$ normal, $1=$ equivocal, $2=$ moderate, $3=$ severe, $4=$ absent). The disagreement in defect score was resolved by consensus. Segments with a defect score of 2 or more 
TABLE 1. Patient characteristics

\begin{tabular}{lc}
\hline \multicolumn{1}{c}{ Parameter } & Value \\
\hline Gender & \\
$\quad$ Male & 19 \\
Female & 2 \\
Age (y) & $63 \pm 8(55-72)$ \\
History of MI & 10 \\
Diabetes & 7 \\
No. of vessels diseased & \\
3 & 15 \\
2 & 6 \\
LVEF (\%) & $49 \pm 6(35-55)$ \\
& \\
Anatomic distribution of CAD & No. of patients/degree \\
Left main & of stenosis* \\
LAD & $6 / 21(60 \%-90 \%)$ \\
Diagonals & $21 / 21(60 \%-100 \%)$ \\
LCx & $7 / 21(50 \%-95 \%)$ \\
Obtuse marginals & $15 / 21(60 \%-100 \%)$ \\
RCA & $10 / 21(60 \%-100 \%)$ \\
PDA & $19 / 21(50 \%-100 \%)$ \\
& $6 / 21(50 \%-90 \%)$
\end{tabular}

Chronic total occlusions

No. of patients

Any vessel 10/21

LAD $\quad 5 / 21$

LCX/OMs $\quad 4 / 21$

RCA 6/21

$M I$, Myocardial infarction; $C A D$, coronary artery disease; $L A D$, left anterior descending coronary artery; $L C x$, left circumflex coronary artery; $R C A$, right coronary artery; $P D A$, posterior descending artery (arising from right coronary artery in all patients); $O M$, obtuse marginal branches; $L V E F$, left ventricular ejection fraction. *Number of patients with disease presence in the reference vessel (defined as stenosis $\geq 50 \%$ of the lumen diameter) and range of stenosis percentage (inside parentheses).

were defined as abnormal and the total MIBG defect score (TDS) was derived as the sum of all segments.

Furthermore, polar maps were derived from the short-axis SPECT images using dedicated computer software and were compared with those of age-matched normal subjects on a pixel-by-pixel basis. Regions of reduced tracer uptake were defined using a threshold of $50 \%$ of the maximum and defect sizes were measured for the whole left ventricle as a percentage of the size of the whole myocardium, which was measured as the volume of the consecutive short-axis slices from base to apex. Regional defect size was also calculated for the 3 regions corresponding to the 3 coronary arteries.

\section{Statistical Analysis}

Analyses were performed with SPSS version 16 software (SPSS, Inc, Chicago, Ill). All data were expressed as mean values \pm standard deviation, with selected range values. Repeated-measurements analysis of variance with the Bonferroni adjustment was performed to compare TDS, H/M ratio, total and regional defect size, and QTc values between pre-CABG (condition A), 1 week after CABG (condition B), and 6 months after CABG (condition C) conditions. The Maulchy test of sphericity was used to test the hypothesis that the variances of differences of the parameters evaluated were equal among the 3 conditions. When the Maulchy test statistic was less than .05 , the degrees of freedom in the univariate testing of the effect of serial imaging in the change of the individual parameters evaluated were corrected with the Greenhouse-Geisser correction. Linear regression analysis was used to evaluate the relationship between MIBG uptake and H/M ratio values in the early and late post-CABG imaging with maximum postprocedural myocardial enzyme levels and aortic crossclamp times. Binary logistic regression analysis was used for evaluation of (1) relationships between CRRAEs and MIBG imaging parameters and (2) variables related to lack of improvement in the MIBG defect size/score in the final imaging study compared with baseline. Goodness-of-fit for the latter analysis was examined using the Hosmer-Lemeshow test.

\section{RESULTS}

All patients underwent successful coronary artery revascularization, with uncomplicated weaning from cardiopulmonary bypass (CPB), except for 1 case of successful open chest cardiac defibrillation for ventricular fibrillation occurring immediately after restoration of circulation. The recovery of this patient after that event was unremarkable. Seven patients received 4 grafts, eight 3 grafts and six 2 grafts. Complete revascularization was achieved in 13 patients. The mean duration of CPB was $115 \pm 33$ minutes (range, 65-189 minutes) and the mean aortic crossclamp time was $64 \pm 30$ minutes (range, 31-124 minutes). On completion of the procedure, patients were transferred initially to the ICU, where they remained for a median duration of 2 days on temporary low-dose combined inotropic support (dobutamine and dopamine), according to standard ICU policy. Inotrope administration was terminated on ICU discharge. During ICU stay, 2 patients required a short period of intensive circulatory support to reverse fluid challenge-resistant hypotension associated with oliguria $\left(<0.5 \mathrm{~mL} \cdot \mathrm{kg}^{-1} \cdot \mathrm{h}^{-1}\right)$. One patient required a purely pharmacologic treatment (vassopressors and high dobutamine dose) and the other a combination of pharmacologic and intra-aortic balloon counterpulsation support.

Temporal elevation of myocardial enzymes indicative of periprocedural myocardial necrosis was observed in 4 patients, but none of them fulfilled criteria for CABG-related MI. Enzyme elevation started on the first postoperative day in every case and lasted for a median duration of 84 hours for creatine kinase $\mathrm{MB}$, with median maximum values for troponin $\mathrm{I}$ and creatine kinase MB being $39 \pm 31 \mu \mathrm{g} / \mathrm{L}$ and $60 \pm 59 \mathrm{U} / \mathrm{L}$, respectively. Two patients had atrial fibrillation on the second postoperative day, which was successfully terminated with intravenous use of amiodarone. These patients were excluded from analysis of QTc and heart rate statistics. All patients were discharged home after a mean duration of $9 \pm 2$ days.

Biochemical parameters with possible implications in the MIBG imaging results on the day of the second MIBG imaging sequence were as follows: hemoglobin, $110 \pm 20 \mathrm{~g} / \mathrm{L}$; white blood cell count, $8.7 \pm 2.3 \cdot 10^{9} / \mathrm{L}$; potassium, $4.6 \pm$ $0.4 \mathrm{mmol} / \mathrm{L}$; and glucose, $7.7 \pm 2 \mathrm{mmol} / \mathrm{L}$.

The Maulchy test statistic was less than .05 for total defect size and score and regional defect size for the RCA and LCx territories (where the Greenhouse-Geisser correction was used) and nonsignificant for QTc, H/M ratio, and regional defect size for the LAD territory. In univariate 
TABLE 2. MIBG scanning parameters of global assessment

\begin{tabular}{|c|c|c|c|c|c|c|c|}
\hline \multirow[b]{2}{*}{ Parameter } & \multirow[b]{2}{*}{ Pre (A) } & \multirow{2}{*}{$\begin{array}{c}\text { One week } \\
\text { after (B) }\end{array}$} & \multirow{2}{*}{$\begin{array}{c}\text { Six months } \\
\text { after }(\mathrm{C})\end{array}$} & \multirow[b]{2}{*}{$P$ value } & \multirow[b]{2}{*}{ Mean difference } & \multicolumn{2}{|c|}{$\mathbf{9 5} \%$ CI for difference } \\
\hline & & & & & & Upper bound & Lower bound \\
\hline \multirow[t]{3}{*}{$\mathrm{H} / \mathrm{M}$ ratio } & $1.9 \pm 0.3$ & $1.5 \pm 0.4$ & $1.9 \pm 0.2$ & A vs B: $<.0001$ & A-B: $.39 \pm .07$ & .21 & .57 \\
\hline & & & & A vs C: NS & A-C: $.02 \pm .05$ & -.12 & .17 \\
\hline & & & & B vs C: $<.0001$ & B-C: $-.37 \pm .06$ & -.54 & -.19 \\
\hline \multirow[t]{3}{*}{ Total defect score } & $24 \pm 5$ & $32 \pm 9.5$ & $23 \pm 6$ & A vs B: $<.0001$ & A-B: $-8.0 \pm 1.54$ & -12.0 & -4.0 \\
\hline & & & & A vs C: NS & A-C: $.57 \pm .7$ & -1.25 & 2.4 \\
\hline & & & & B vs C: $<.0001$ & B-C: $8.6 \pm 1.1$ & 5.67 & 11.5 \\
\hline \multirow[t]{3}{*}{ Total defect size $(\%)$} & $37 \pm 8.7$ & $49.5 \pm 20.4$ & $36.3 \pm 12.6$ & A vs B: .004 & A-B: $-12.5 \pm 3.3$ & -21.3 & -3.8 \\
\hline & & & & A vs C: NS & A-C: $.6 \pm 1.8$ & -4.2 & 5.4 \\
\hline & & & & B vs C: $<.0001$ & B-C: $13.1 \pm 2.6$ & 6.2 & 20.1 \\
\hline
\end{tabular}

The mean differences are significant at the .05 level, whereas the $P$ values and the confidence intervals are adjusted for multiple comparisons with the Bonferroni correction. MIBG imaging parameters of global evaluation were measured before and after coronary artery bypass grafting (designated A, B, and C). MIBG, Meta-iodobenzyl-guanide; $C I$, confidence interval; $H / M$, heart/mediastinum; $N S$, not significant, that is, larger than .05 .

testing, serial imaging had significant interaction with every parameter evaluated, except for the regional defect size in the RCA territory.

At 1 week after CABG, patients had a significantly higher TDS and total defect size and a significantly decreased H/M ratio compared with the pre-CABG corresponding values. MIBG uptake was reduced in all but 4 patients.

Six months after surgery, the values of the aforementioned indices were not significantly different from the pre-CABG values. MIBG uptake at 6 months, though, was increased in only 9 patients with regard to the preCABG imaging. Hence, an almost homogeneous and significant worsening of all MIBG parameters was observed initially after $\mathrm{CABG}$, with an equally homogeneous improvement in these values in the delayed vs the early post-CABG scans. However, the 6-month post-CABG improvement was very variable with respect to the baseline status, leading to nonsignificant differences of the MIBG imaging parameters between $\mathrm{A}$ and $\mathrm{C}$ conditions. The latter is reflected in the wide $95 \%$ confidence intervals of the mean difference in TDS and size between conditions A and $\mathrm{C}$, in contrast to the narrow intervals observed in the comparisons between $\mathrm{A}$ and $\mathrm{B}$ and $\mathrm{B}$ and $\mathrm{C}$. MIBG imaging parameters of global evaluation $(\mathrm{H} / \mathrm{M}$ ratio, TDS, and total defect size) before and after CABG are presented in Table 2, whereas Figure 1 is a graphic representation of the temporal trends observed in total defect size and $\mathrm{H} / \mathrm{M}$ ratio during sequential MIBG imaging for each of the patients studied.

In terms of regional evaluation, the temporal pattern of early decrease and late increase in CSNF was observed in all pairwise comparisons between the 3 territories studied. All these changes were statistically significant with the exception of change from A to B condition for RCA. At 6 months no differences in MIBG size were documented
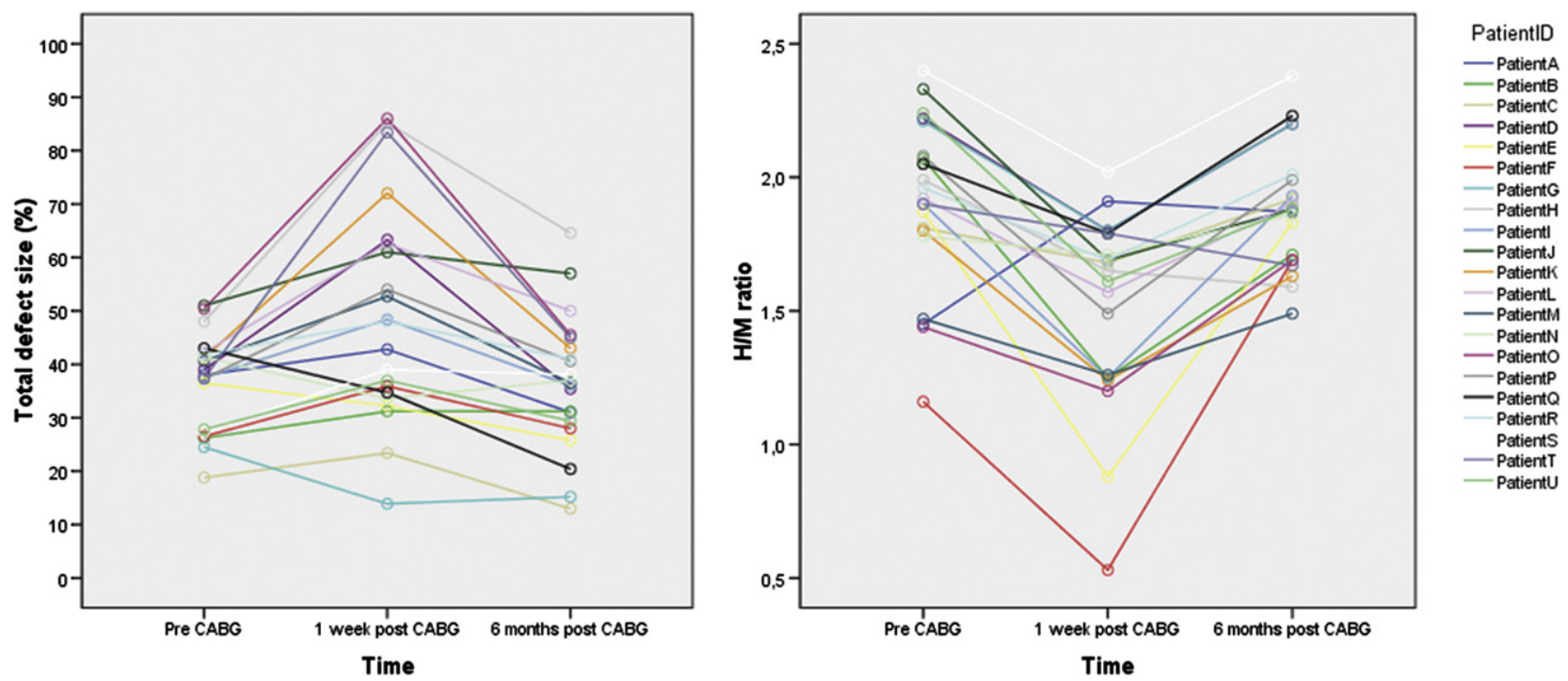

FIGURE 1. Graphic representation of the serial changes in total defect size (left panel) and heart/mediastinum (H/M) ratio (right panel) during sequential meta-iodobenzyl-guanide imaging for each of the patients studied. $C A B G$, Coronary artery bypass grafting. 
TABLE 3. Local assessment of percent defect size according to the polar maps (bull's eye) by vessel territory

\begin{tabular}{|c|c|c|c|c|c|c|c|}
\hline \multirow[b]{2}{*}{ Vessel territory } & \multirow[b]{2}{*}{ Before (A) } & \multirow{2}{*}{$\begin{array}{c}\text { One week } \\
\text { after (B) }\end{array}$} & \multirow{2}{*}{$\begin{array}{c}\text { Six months } \\
\text { after (C) }\end{array}$} & \multirow[b]{2}{*}{$P$ value } & \multirow[b]{2}{*}{ Mean difference } & \multicolumn{2}{|c|}{$95 \%$ CI for difference* } \\
\hline & & & & & & Upper bound & Lower bound \\
\hline \multirow[t]{3}{*}{$\overline{L A D}$} & $36.1 \pm 10.6$ & $48.1 \pm 18.8$ & $30.2 \pm 8.5$ & A vs B: .012 & A-B: $-11.96 \pm 3.68$ & -21.58 & -2.34 \\
\hline & & & & B vs $\mathrm{C}:<.001$ & B-C: $17.87 \pm 3.29$ & 9.27 & 26.47 \\
\hline & & & & A vs C: NS & A-C: $5.91 \pm 2.88$ & -1.61 & 13.43 \\
\hline \multirow[t]{3}{*}{ RCA } & $44.5 \pm 23.1$ & $48.0 \pm 26.3$ & $40.5 \pm 24.1$ & A vs B: NS & A-B: $-3.51 \pm 5.48$ & -17.85 & 10.82 \\
\hline & & & & B vs C: .001 & B-C: $7.505 \pm 1.7$ & 3.05 & 11.96 \\
\hline & & & & A vs C: NS & A-C: $4 \pm 4.98$ & -9.01 & 17.0 \\
\hline \multirow[t]{3}{*}{$\mathrm{LCx}$} & $25.6 \pm 24.1$ & $61.8 \pm 27.9$ & $39.5 \pm 17.0$ & A vs $\mathrm{B}:<.001$ & A-B: $-36.2 \pm 5.33$ & -50.1 & -22.26 \\
\hline & & & & B vs C: $<.001$ & B-C: $22.32 \pm 3.13$ & 14.14 & 30.5 \\
\hline & & & & A vs C: .050 & A-C: $-13.86 \pm 5.31$ & -27.74 & 0.02 \\
\hline
\end{tabular}

$C I$, Confidence intervals; $L A D$, left anterior descending coronary artery; $R C A$, right coronary artery; $L C x$, left circumflex coronary artery; $N S$, not significant, that is, larger than .05 . *The mean differences are significant at the .05 level, and the $P$ values and the confidence intervals are adjusted for multiple comparisons with the Bonferroni correction.

compared with baseline for all coronary artery territories. Table 3 demonstrates the local assessment of percent defect size by coronary artery territory, according to the polar maps.

QTc values before CABG ( $422 \pm 41 \mathrm{~ms})$ had no statistically significant differences with the values recorded either at 1 week $(420 \pm 33 \mathrm{~ms})$ or at 6 months $(424 \pm 36 \mathrm{~ms})$ after CABG.

In linear regression analysis, no correlation was found between TDS and total defect size or $\mathrm{H} / \mathrm{M}$ ratio at either 1 week or 6 months after $\mathrm{CABG}$, with aortic crossclamp time or maximum postprocedural myocardial enzyme levels.

At the end of the study, 1 hospitalization but no fatal events were observed. Hospitalization resulted from an acute coronary syndrome at 5 months after CABG; the culprit vessel was the posterolateral ventricular branch of the
RCA, which was not revascularized owing to technical difficulties. In addition, 2 more patients were discovered with asymptomatic atrial fibrillation at scheduled post-CABG office visits and underwent successful electric cardioversion after appropriate anticoagulation as day cases at the hospital. All patients were in sinus rhythm at the end of the study and a total number of 7 CRRAEs were recorded during the study's follow-up. Further follow-up data were available for a median duration of 590 days after the end of the study. No late deaths, sustained arrhythmias, or major adverse cardiac events were observed during this period.

MIBG imaging parameters, performance of complete revascularization, prior MI, periprocedural myocardial necrosis, age, diabetes, left ventricular ejection fraction, and CPB
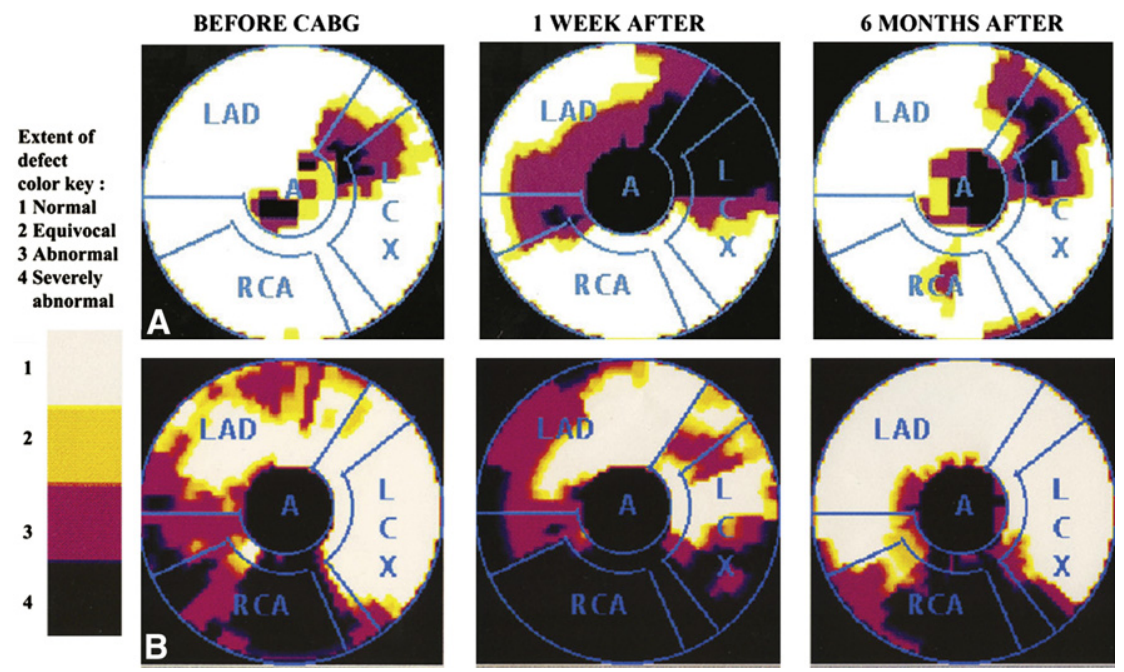

FIGURE 2. Polar maps of 2 representative patients, depicting meta-iodobenzyl-guanide $(M I B G)$ uptake before and after coronary artery bypass grafting $(C A B G)$. Tracer uptake before (left column), 1 week after (middle column), and 6 months after (right column) CABG surgery is shown. Patient A (top) is a 54-year-old woman with 2-vessel coronary artery disease $(C A D)$ who received 2 venous grafts. Reversible reduction of MIBG uptake at 1 week after surgery is clearly demonstrated. Patient B (bottom) is a 67-year-old man with 3-vessel CAD who underwent successful CABG surgery with 1 arterial and 2 venous grafts. Note the significant reduction of MIBG uptake immediately after surgery and the improvement of MIBG uptake 6 months later, at levels even higher than the ones before surgery. A, Apex; $L A D$, left anterior descending coronary artery; $L C x$, left circumflex coronary artery; $R C A$, right coronary artery. 
time were used as independent variables in a binary logistic regression analysis with CRRAEs as the dependent variable. Among them, only the difference in MIBG total score between the first and the second imaging was significantly related to CRRAEs $(P=.028$; hazard ratio, $1.155 ; 95 \%$ confidence intervals for hazard ratio, 1.016-1.314). Using the same type of analysis and the same variables including occurrence of postprocedural acute coronary syndrome, no variables were found to be associated with lack of improvement in MIBG score or size in the last imaging.

Figure 2 depicts the MIBG uptake in 2 representative patients before surgery and 1 week and 6 months after surgery.

\section{DISCUSSION}

Despite the broad spectrum of surgical-interventional procedures associated with reversible or fixed reduction in CSNF and abnormal MIBG uptake, ${ }^{12-15}$ insufficient data exist to support these phenomena after $\mathrm{CABG}^{8}$ On the other hand, Momose and associates ${ }^{16}$ did not demonstrate CABG-induced reduction in $\mathrm{H} / \mathrm{M}$ ratio in a very small series of patients undergoing MIBG imaging before and late after the procedure. These 7 patients served as a control group for the evaluation of CSNF changes after surgical repair of ascending aortic aneurysm. To our knowledge, this is the first study to demonstrate that CABG surgery is related to significant and reversible reduction in CSNF, which is clinically important.

Concern about possible confounding effects of using beta-blockers or the presence of patients with diabetes in the population studied is not justified. The conventional beta-blockers used have limited effects in MIBG uptake and biodistribution, ${ }^{17}$ whereas the comparative nature of the study under the same treatment environment attenuates any effect that beta-blockers would have on an isolated MIBG imaging study. Furthermore, cardiac autonomic dysfunction in patients with longstanding diabetes is permanent. ${ }^{18}$ Hence, neither special analysis would be justified, nor would different results be expected for the diabetic patients of our study.

There are 2 possible mechanisms responsible for the reduction of cardiac MIBG uptake: (1) peripheral sympathetic nerve hyperactivity and (2) direct or indirect cardiac sympathetic nerve damage caused by the CABG procedure.

Regarding the first mechanism, reduced MIBG uptake may be seen with high circulating catecholamine levels as a result of competition for the type 1 uptake mechanism. ${ }^{17}$ Consequently, a state of peripheral adrenergic hyperactivity, related to the physical and emotional postoperative stress, could account for a decreased cardiac MIBG uptake early after the $C A B G$ procedure. Still, in a generalized state of increased sympathetic tone serious enough to cause competition of peripheral adrenergic nerve endings for MIBG uptake, QTc should have also been influenced.
With respect to the other 2 possible mechanisms, the following facts should be noted. All the revascularization procedures using vein grafts had the potential for direct injury to the cardiac sympathetic nerve plexus that courses along the base of the great arteries, by adventitia abrasion or by punching out the wall in the ascending aorta, during proximal graft anastomosis. Furthermore, indirect damage to adrenergic nerve endings from the neurotoxic effects of periprocedural ischemia (iatrogenic or induced by circulatory arrest), cardioplegic solutions, ${ }^{19,20}$ and hypothermia ${ }^{21,22}$ could play a major role to the findings of this study.

Early after $C A B G$ the combined effect of the above neurotoxic factors was predominant, leading to significant deterioration of MIBG imaging parameters despite the revascularization-induced improvement in blood supply. As the power of this effect progressively diminished with time, restoration of MIBG imaging parameters was observed but not improvement compared with their baseline values. Possibly part of this negative effect is still active enough to counterbalance the beneficial effect of revascularization even late after CABG. This lack of improvement supports analogous findings by Hayashi and colleagues ${ }^{8}$ and deserves special mention. We failed to identify a specific variable significantly related to this paradox, a fact that we believe supports the multifactorial cause of this phenomenon and begs for further study.

Although no concurrent perfusion studies were performed and complete revascularization was not uniformly achieved, there can be no argument that significant improvement in blood supply was achieved and that revascularization was practically the only beneficial intervention for CSNF between the first and the last MIBG imaging. Improved medical therapy after surgery, limited to increased postprocedural statin use, had no impact in the apparent reinnervation inasmuch as statins have never been documented to affect CSNF.

Finally and most important, the observed sympathetic denervation was found to be clinically relevant. Alterations in CSNF are implicated in arrhythmogenesis and myocardial dysfunction, and we demonstrated that the CABG-induced reduction in CSNF is related to major contributors in postprocedural morbidity and mortality, namely, arrhythmias ${ }^{23}$ and operation-induced myocardial stunning. ${ }^{24}$

\section{Limitations}

There are a number of limitations in our study.

First, we did not perform simultaneous myocardial perfusion imaging along with MIBG imaging, which could help in clarifying the cause of our findings, as well as the exact mechanism for the lack of uniform improvement in postprocedural MIBG uptake compared with baseline status. Evidence exists, though, for a discrepancy between MIBG and ${ }^{201} \mathrm{Tl}$ SPECT imaging findings in the early postoperative period, after successful surgical 
revascularization. ${ }^{8}$ Hence, the effect of this limitation is probably attenuated.

Second, we did not use a more direct means of sympathetic tone assessment. Blood catecholamines could be measured instead of QTc, with its inherent limitations as a method of evaluating autonomic nervous system status, but plasma levels of norepinephrine in heart diseases rarely, if ever, exceed levels that can inhibit MIBG uptake. ${ }^{25}$

In addition, we used only delayed images for MIBG, while in patients with previous MI we did not compare MIBG findings between infarcted and noninfarcted regions.

Finally, this study was not properly controlled, although it is highly unlikely for either normal persons or subjects with CAD to have significant, spontaneous changes in MIBG imaging parameters with time.

We thank Mrs Anna Koutsimpani for her help in preparing the manuscript.

\section{References}

1. Henneman MM, Bengel FM, van der Wall EE, Knuuti J, Bax JJ. Cardiac neuronal imaging: application in the evaluation of cardiac disease. $J$ Nucl Cardiol. 2008; 15:442-55.

2. Flotats A, Carrió I. Cardiac neurotransmission SPECT imaging. J Nucl Cardiol. 2004;11:587-602.

3. Simula S, Lakka T, Kuikka J, Laitinen T, Remes J, Kettunen R, et al. Cardiac adrenergic innervation within the first 3 months after acute myocardial infarction. Clin Physiol. 2000;20:366-73.

4. Wichter T, Matheja P, Eckardt L, Kies P, Schafers K, Schulze-Bahr E, et al. Cardiac autonomic dysfunction in Brugada syndrome. Circulation. 2002;105:702-6.

5. Kies P, Paul M, Gerss J, Stegger L, Monnig G, Schober O, et al. Impaired cardiac sympathetic innervation in symptomatic patients with long QT syndrome. Eur J Nucl Med Mol Imaging. 2011;38:1899-907.

6. Nagamatsu H, Momose M, Kobayashi H, Kusakabe K, Kasanuki H. Prognostic value of 123I-metaiodobenzylguanidine in patients with various heart diseases. Ann Nucl Med. 2007;21:513-20.

7. Verberne HJ, Brewster LM, Somsen GA, van Eck-Smit BL. Prognostic value of myocardial 123I-metaiodobenzylguanidine (MIBG) parameters in patients with heart failure: a systematic review. Eur Heart J. 2008;29:1147-59.

8. Hayashi I, Makuuchi H, Naruse Y, Kobayashi T, Yamamoto T, Hariya A, et al. The clinical value of MIBG myocardial SPECT before and after CABG-comparison with TL myocardial SPECT. Nippon Kyobu Geka Gakkai Zasshi. 1995;43:1670-4.

9. Thygesen K, Alpert JS, White HD. Universal definition of myocardial infarction. J Am Coll Cardiol. 2007;50:2173-95.
10. Browne KF, Zipes DP, Heger JJ, Prystowsky EN. Influence of the autonomic nervous system on the Q-T interval in man. Am J Cardiol. 1982;50:1099-103.

11. Veglio M, Maule S, Matteoda C, Quadri R, Valentini M, Pecchio O, et al. Use of corrected QT interval in autonomic function testing: assessment of reproducibility. Clin Auton Res. 1996;6:309-12.

12. Kondo C, Nakazawa M, Momma K, Kusakabe K. Sympathetic denervation and reinnervation after arterial switch operation for complete transposition. Circulation. 1998;97:2414-9.

13. Mabuchi M, Imamura M, Kubo N, Morita K, Noriyasu K, Tsukamoto T, et al. Sympathetic denervation and reinnervation after the maze procedure. $\mathrm{J} \mathrm{Nucl}$ Med. 2005;46:1089-94.

14. Beek JF, van der Sloot JA, Huikeshoven M, Verberne HJ, van Eck-Smit BL, van der Meulen J, et al. Cardiac denervation after clinical transmyocardial laser revascularization: short-term and long-term iodine 123-labeled metaiodobenzylguanide scintigraphic evidence. J Thorac Cardiovasc Surg. 2004; 127:517-24.

15. Buendia-Fuentes F, Almenar L, Ruiz C, Vercher JL, Sanchez-Lazaro I, MartinezDolz L, et al. Sympathetic reinnervation 1 year after heart transplantation, assessed using iodine-123 metaiodobenzylguanidine imaging. Transplant Proc. 2011;43:2247-8.

16. Momose M, Kobayashi H, Ikegami H, Nagamatsu H, Sakomura Y, Aomi S, et al. Total and partial cardiac sympathetic denervation after surgical repair of ascending aortic aneurysm. J Nucl Med. 2001;42:1346-50.

17. Shapiro B, Gross MD. Radiochemistry, biochemistry, and kinetics of 131I-metaiodobenzylguanidine (MIBG) and 123I-MIBG: clinical implications of the use of 123I-MIBG. Med Pediatr Oncol. 1987;15:170-7.

18. Schnell O, Muhr D, Weiss M, Kirsch CM, Haslbeck M, Tatsch K, et al. Threeyear follow-up on scintigraphically assessed cardiac sympathetic denervation in patients with long-term insulin-dependent (type I) diabetes mellitus. J Diabetes Complications. 1997;11:307-13.

19. Bengtsson L, Henze A, Farnebo LO. Decreased liberation of noradrenaline from the heart following cold crystalloid cardioplegia. Scand J Thorac Cardiovasc Surg. 1991;25:147-50.

20. Murphy DA, Armour JA. Influences of cardiopulmonary bypass, temperature, cardioplegia, and topical hypothermia on cardiac innervation. $J$ Thorac Cardiovasc Surg. 1992;103:1192-9.

21. Kitagawa H, Akiyama T, Yamazaki T. Effects of moderate hypothermia on in situ cardiac sympathetic nerve endings. Neurochem Int. 2002;40:235-42.

22. Tokunaga S, Imaizumi T, Fukae K, Nakashima A, Hisahara M, Tominaga R, et al. Effects of hypothermia during cardiopulmonary bypass and circulatory arrest on sympathetic nerve activity in rabbits. Cardiovasc Res. 1996;31:769-76.

23. Kaw R, Hernandez AV, Masood I, Gillinov AM, Saliba W, Blackstone EH. Shortand long-term mortality associated with new-onset atrial fibrillation after coronary artery bypass grafting: a systematic review and meta-analysis. $J$ Thorac Cardiovasc Surg. 2011;141:1305-12.

24. Birjiniuk V. Patient outcomes in the assessment of myocardial injury following cardiac surgery. Ann Thorac Surg. 2001;72:S2208-12; discussion S2212-3, S2267-70.

25. Glowniak JV. Cardiac studies with metaiodobenzylguanidine: a critique of methods and interpretation of results. J Nucl Med. 1995;36:2133-7. 
TABLE E1. Patients' medications during MIBG imaging, with the relative number of patients by medication used

\begin{tabular}{lccc}
\hline Time point drugs & $\begin{array}{c}\text { Before } \\
\text { CABG (A) }\end{array}$ & $\begin{array}{c}\text { One week } \\
\text { after CABG (B) }\end{array}$ & $\begin{array}{c}\text { Six months } \\
\text { after CABG (C) }\end{array}$ \\
\hline Beta-blockers & 19 & 19 & 19 \\
Aspirin & 21 & 21 & 21 \\
Statin & 12 & 12 & 16 \\
Nitrates & 15 & 3 & 1 \\
PPIs & 10 & 17 & 15 \\
KCl supplements & 5 & 11 & 4 \\
Analgesics & 0 & 18 & 0 \\
Metformin & 5 & 5 & 5 \\
Sulfonylureas & 4 & 4 & 4 \\
Others* & 6 & 7 & 5 \\
\hline
\end{tabular}

$M I B G$, Meta-iodobenzyl-guanide; $C A B G$, coronary artery bypass grafting; $P P I s$, proton pump inhibitors. *Non-QT prolonging (according to: www.qtdrugs.org) or MIBG uptake-affecting drugs (according to Solanki KK, Bomanji J, Moyes J, Mather SJ, Trainer PJ, Britton KE. A pharmacological guide to medicines which interfere with the biodistribution of radiolabeled meta-iodobenzylguanidine (MIBG). Nucl Med Commun. 1992;13:513-21). 